\title{
SSSC Forum Delineates Future Role of Materials Research in U.S. Scientific Policy
}

"Materials in a New Era" was the theme of the 1999 Solid State Sciences Committee (SSSC) Forum held February 16-17 at the National Academy of Sciences in Washington, DC. For two days, leaders from the materials research community met with agency heads and policymakers to discuss the past and future role of materials research in the United States and beyond. The tone of the meeting was considerably more upbeat than at the last Forum held three years ago when the funding picture for science was very bleak. In the interim, the federal funding picture for scientific research has stabilized and improved awareness of the value of sustained investment in research has increased in at least some quarters in Washington, and industrial support for the physical sciences has stabilized. With such relatively good news, the community could become complacent about being recognized as an invaluable contributor to the U.S. and world economy. A major theme of the Forum was that the materials research community cannot afford complacency.

Laura Rodriguez, a staff member in the office of Representative Vern Ehlers (RMich.), kicked off the conference with an overview of the recently issued document "Unlocking Our Future: Toward a New National Science Policy." This is the report of a House of Representatives study headed by Rep. Ehlers aimed at developing a national science policy that will be appropriate for the 21st century. The study finds that the federal government, scientists, and educators must address several issues: (1) The United States' science policy is outdated; (2) the American public does not understand science and its practice; and (3) scientists are politically clueless. The report makes four major recommendations: (1) Continue to push the boundaries of the scientific frontier by supporting interdisciplinary research, maintaining a balanced research portfolio, and funding more innovative "risk-taking" projects; (2) support private research efforts, an essential component of a healthy U.S. research and development (R\&D) portfolio, by encouraging young, start-up companies, making the R\&D tax credit permanent, streamlining regulations, and pursuing and developing effective partnerships; (3) increase efforts in education at all levels including pre-school to graduate school; research on curricula and education; address issues of teacher training, recruitment, and retention; provide for a more diversi- fied graduate experience; and increase public outreach; and (4) strengthen the relationship between science and the society that supports it through improved communication among scientists, journalists, and the public and by engaging the scientific community in helping society make good decisions.

The interdependence of different fields of research was addressed by a number of representatives of federal agencies. Artie Bienenstock, Associate Director for Science in the Office of Science and Technology Policy emphasized the Clinton Administration's unequivocal commitment to maintaining leadership across the frontiers of scientific knowledge. Technology and the underlying science in many fields are responsible for more than $50 \%$ of the increases in productivity that researchers have enjoyed over the last 50 years. The various branches of science are truly interdependent-progress in one field depends on advances in many other areas. As an example, Bienenstock pointed to CAT scans, one of the mainstays of medical diagnostics, asking why it took so long after the discovery of $x$-rays for the technology to develop. Progress in many fields was needed to make the technology a reality: solid-state physics and engineering to enable the computers that control the instrument and collect and analyze the data, materials science to provide the $x$ ray detectors, and mathematics and computer science for the algorithms to reconstruct the image from the raw data. CAT scans would be unknown if any of these were missing.

Marvin Cassman, Director, National Institute of General Medical Sciences, further embroidered the theme of interdependence by discussing the multidisciplinary nature of research at major facilities such as synchrotrons and neutron sources. In the United States, most such facilities are funded by agencies with major responsibility for condensed matter and materials research. Biological research, however, is finding increasing need for the facilities, so that it now accounts for a significant fraction of all work being carried out in those venues. Appropriate cooperation among these communities and the agencies that fund them will be essential to the continued viability of these important and extremely costly facilities.

Martha Krebs, Director of the Department of Energy Office of Science, presented the view from DOE. The FY00 budget request for the Office of Science is $\$ 184 \mathrm{M}$ greater than the FY99 budget. This increase is largely for construction of the Spallation Neutron Source and for the Scientific Simulation Initiative (SSI), an interagency initiative that will bring teraflop-scale computing to bear on a number of problems including global systems, combustion, and basic science (which may include materials). Krebs identified a number of future directions and opportunities in materials research including neutron scattering, materials at high magnetic fields, granular materials, complex materials, and high-temperature superconductors and other vortex matter. (For more information on SSI, see the April 1999 issue of MRS Bulletin, page 10.)

Hans Mark, Director for Defense Research and Engineering in the Department of Defense, suggested four new science and technology topics that the Defense Science Board should be considering and invited the scientific community to suggest others. The ones he suggested were (1) "strange" molecules, that is, forms of carbon or hyperbranched molecules; (2) software development, especially new techniques for producing software such as genetic algorithm development and application and automation of software development; (3) high-power electrical devices; and (4) predictive chaos theory/nonlinear dynamics and its applicability to national security.

Venkatesh Narayanamurti, Dean of Applied Science, Harvard University and Chair of the Committee on Condensed Matter and Materials Physics (CMMP), gave an overview of the report of the CMMP Committee scheduled for publication this Spring. His talk was followed by more extensive discussion of some of the findings of the committee, including areas for future research, the research climate for CMMP, and the role of facilities. The report and its findings and recommendations will be the subject of an upcoming Public Affairs Forum.

Thomas P. RusSell Julia M. Phillips

Thomas P. Russell is professor of polymer science and engineering at the University of Massachusetts, Amherst and is the chair of the Solid State Sciences Committee.

Julia M. Phillips is a member of the Solid State Sciences Committee and served on the Committee on Condensed Matter and Materinls Physics. 